\title{
A strategy to optimize radiation exposure for non-contrast head CT: comparison with the Japanese diagnostic reference levels
}

\author{
Kanako K. Kumamaru ${ }^{1}$ - Yosuke Kogure ${ }^{1} \cdot$ Michimasa Suzuki $^{1} \cdot$ Masaaki Hori $^{1}$ • \\ Atsushi Nakanishi $^{1} \cdot$ Koji Kamagata $^{1} \cdot$ Akifumi Hagiwara $^{1} \cdot$ Christina Andica $^{1}$. \\ Keiken $\mathrm{Ri}^{2} \cdot$ Naoyoshi Houshido ${ }^{1} \cdot$ Shigeki Aoki $^{1}$
}

Received: 21 February 2016 / Accepted: 1 April 2016 / Published online: 20 April 2016

(C) Japan Radiological Society 2016

\begin{abstract}
Purpose To describe how we performed a protocol review, analyzed data, identified opportunities to reduce radiation exposure, and then implemented a new imaging protocol for non-contrast adult head $\mathrm{CT}$ at our institution with reduced radiation exposure, using the Japanese diagnostic reference levels (DRLs) as the reference.

Methods After analyzing the CT dose index $\left(\mathrm{CTDI}_{\mathrm{vol}}\right)$ and dose length product (DLP) in all non-contrast adult head CT examinations performed during a 3-month period (September to November 2015) in order to identify a specific protocol that contributed to the above-DRL-level radiation exposure observed for non-contrast adult head CT at our institution, phantom studies with objective and subjective image quality analyses were performed to develop a new imaging protocol. After implementing the new proto$\mathrm{col}$, its feasibility was evaluated in terms of radiation exposure, prevalence of significant disease detection, and subjective image quality.

Results The review of 2040 examinations revealed that a helical protocol $\left(\mathrm{CTDI}_{\mathrm{vol}}=93.4 \mathrm{mGy}\right)$ with one of four CT scanners mainly contributed to the aboveDRL-level radiation exposure (mean DLP at this scanner $=1401.2 \mathrm{mGy} \mathrm{cm}$ ) in non-contrast adult head CT at our institution. To replace this protocol, the phantom study identified a wide-volume scan using $120 \mathrm{kVp}, 350 \mathrm{mAs}$, a 4-cm detector, a slice thickness of $5 \mathrm{~mm}$, and a $\mathrm{CTDI}_{\mathrm{vol}}$ of
\end{abstract}

Kanako K. Kumamaru

k-kumamaru@juntendo.ac.jp

1 Department of Radiology, Juntendo University, 2-1-1 Hongo, Bunkyo-ku, Tokyo 113-8421, Japan

2 School of Medicine, Juntendo University, 2-1-1 Hongo, Bunkyo-ku, Tokyo 113-8421, Japan
$69.8 \mathrm{mGy}$ as a new protocol that yielded comparable image quality to the existing protocol. After the implementation of the new protocol, the overall mean DLP reduced to $1365 \mathrm{mGy} \mathrm{cm}$ without any apparent degradation of image quality. No significant decrease in the prevalence of significant findings after protocol revision was noted.

Conclusion We report the successful implementation of a new protocol with reduced radiation exposure for non-contrast head CT examinations.

Keywords Computed tomography · Radiation exposure · Diagnostic reference level

\section{Introduction}

Diagnostic reference levels (DRLs) were first advocated by the International Commission on Radiological Protection (ICRP) in the 1990s in order to optimize the radiation dose delivered to patients in the course of diagnostic and/ or therapeutic procedures [1]. A DRL is defined as "a form of investigation level, applied to an easily measured quantity, usually the absorbed dose in the air or tissue-equivalent material at the surface of a simple standard phantom or a representative patient" [1]. DRLs are generally defined as the 75th percentile of the typical dose distribution for patient or phantom measurements [2], so DRLs are "reference" levels rather than dose limits, and they do not help distinguish between good and poor medical practice. If the typical dose used at a facility exceeds the DRL without good clinical reason, the possibility of reducing the mean radiation dose should be seriously pursued [2].

In response to increased concern about overall medical radiation exposure in Japan-especially the radiation exposure due to computed tomography (CT) [3, 4], the Japan 
Network for Research and Information on Medical Exposures (J-RIME) conducted a nationwide survey. Based on the results, Japanese DRLs were established in 2015 [2], which led us to review the mean CT dose index (CTDI) vol $_{\text {ol }}$ (mGy) and dose length product (DLP) (mGy $\mathrm{cm}$ ) at our institution. The mean values for chest, abdomen to pelvic, and cardiac CTs are currently lower than the DRLs; CTDI vol $_{\text {. }}$ was $5 \mathrm{mGy}$ for chest CT, $12 \mathrm{mGy}$ for abdomen to pelvic $\mathrm{CT}$, and $75 \mathrm{mGy}$ for cardiac $\mathrm{CT}$ at our institution, while the DRL was $15 \mathrm{mGy}$ for chest, $20 \mathrm{mGy}$ for abdomen to pelvic, and $90 \mathrm{mGy}_{\text {for cardiac CT. However, the mean CTDI }}$ al and DLP values for non-contrast adult head CT - approximately $90 \mathrm{mGy}$ and $1400 \mathrm{mGy} \mathrm{cm}$, respectively - were higher than the Japan DRLs $\left(85 \mathrm{mGy}\right.$ for CTDI ${ }_{\mathrm{vol}}$ and $1350 \mathrm{mGy} \mathrm{cm}$ for DLP, respectively), so we undertook the project reported in the present work to reduce radiation exposure upon noncontrast head CT in adult patients. Based on the results, radiologists and radiological technologists at our institution reviewed the scan protocols and parameters and discussed how the radiation exposure could be optimized.

The purpose of this report is to describe the concept and strategy employed to optimize radiation exposure in order to accomplish a lower radiation dose with the Japanese DRLs used as the reference, which involved a protocol review, data analysis, the identification of opportunities to reduce exposure levels, and the implementation of a new protocol for non-contrast head $\mathrm{CT}$ to optimize radiation exposure.

\section{Materials and methods}

\section{Study design and population}

All adult patients who underwent non-contrast head CT at our institution-a 1020-bed urban teaching hospital with over 1,000,000 annual outpatients-during a 5-month period (September 2015 to January 2016) were included in this project. We included patients who underwent non-contrast head CT before and after the implementation of the new imaging protocol on 1st December 2015. Retrospective analyses of the data were approved by the institutional review board, who waived the requirement for informed consent.

\section{Review of the existing scan protocols}

At our institution, four CT scanners are currently used for non-contrast head CT examinations. To identify whether a specific protocol or scanner contributed to the above-DRLlevel radiation exposure, we collected the CTDI ${ }_{\mathrm{vol}}$ and DLP data from all non-contrast adult head CT examinations performed during a 3-month period (September-November 2015), which included 2459 examinations.

\section{Disease prevalence in CT examinations performed using the existing protocols}

To obtain background data for the prevalence of significant findings in the examinations before the implementation of the new protocol, a board-certified radiologist with nine years of experience classified the non-contrast adult head CT examinations into four categories based on the radiological findings as follows: category 1 , within normal limits; category 2, abnormalities that do not need follow-up or management, or findings with no interval changes during follow-up; category 3 , findings that need further follow-up; and category 4 , findings that may need treatment or management. The disease prevalence calculated based on those data was employed to assess whether the prevalence of significant findings (categories 3 and 4) changed after the implementation of the new imaging protocol. A similar disease prevalence before and after implementation of the new protocol may indirectly indicate that it does not result in missed diagnoses.

\section{Development of the new imaging protocol based on the phantom study}

As the new protocol was intended for routine use, it had to fulfill the following criteria: the hardware and software that was already in use could also be employed for the new imaging protocol, and it could be performed by radiologic technologists of all skill levels at any time of the day. To identify the protocol that best fulfilled these criteria, we considered quantitative image noise and image quality (contrast-to-noise ratio, CNR), the subjective low-contrast detectability, and the limitations on the spatial resolution by means of a commercially available solid uniformity phantom (Catphan 500, modules CTP515 and CTP528, The Phantom Laboratory, Salem, NY, USA). The CNR is defined as $(A-B) / \mathrm{SD}$, where $A$ is the average CT density expressed in Hounsfield units (HU) of the region of interest (ROI), which is a circle $15 \mathrm{~mm}$ in diameter of $1.0 \%$ of the test object; $B$ is the background; and SD is the standard deviation of the background density. These phantom data were interpreted in accordance with the Catphan 500 reference manual [5]. For all of the protocols tested, a standard soft-tissue kernel was used to evaluate low contrast resolution (CTP515), and a bone kernel was used to evaluate high contrast resolution (CTP528). All the analyses were performed using the OsiriX MD software (v.6.5.2, Pixmeo, Geneva, Switzerland).

\section{Announcement of the new imaging protocol}

After the new imaging protocol had been determined, we created a handout announcing the imminent 
implementation of a new technical protocol for non-contrast adult head CT. This handout explained the reasons for changing the protocol, the target population, and the differences between the old and new techniques; it also included the contact information of the person leading this project. One week before implementing the new imaging protocol, the handout was distributed to the heads of the department, and a meeting was held to notify and explain the protocol change by the radiologists and senior CT technologists.

\section{Implementation of the new imaging protocol}

The new imaging protocol was implemented on 1st December 2015. To ensure that all CT technologists were informed of the protocol changes, and that the protocol change was introduced successfully, the radiologist who led the protocol change (K.K.) remained present at the CT scanner during the first week, in addition to being present at the meeting.

\section{Post-implementation analyses}

We collected data for a 2-month period after implementing the new imaging protocol. The mean DLPs were compared before and after the implementation using the Wilcoxon rank-sum test. We also assessed any change in the prevalence of significant findings (category 3 or 4 ) by the Cochran-Armitage test. In addition, patients who underwent two non-contrast head CTs before and after the protocol change were selected to evaluate subjective imagequality parameters: noise (1, severely degraded images; 2 , moderately degraded images; 3 , minimally degraded images), severity of artifacts (1, severe artifacts affecting interpretation; 2 , artifacts are present but do not negatively affect the interpretation; 3, no artifacts), and gray-white matter differentiation (1, unable to assess; 2 , detectable; 3, well-visualized). Radiologists with 9 and 14 years of experience were blinded to the acquisition parameters, and they visually evaluated all $30 \mathrm{CT}$ studies independently by reviewing axial images on ordinary workstations (Synapse workstation, Fujifilm Medical Systems, Japan). Subjective image quality parameters were scored and compared before and after the change in protocol by means of the Wilcoxon rank-sum test.

\section{Results}

\section{Review of the existing scan protocols}

During the pre-implementation review process, we noticed that cervical spine examinations were sometimes included in head CT examinations, leading to a higher mean DLP.
Accordingly, we excluded these examinations from further analysis, resulting in a final population of 2040 examinations from the vertex to the base of the skull. The mean DLP of these 2040 scans was $1385 \mathrm{mGy} \mathrm{cm}$, which was still higher than the Japanese DRL. The majority of the studies $(90.3 \%)$ were performed by scanner \#1 (Table 1), for which the mean DLP was $1401.2 \mathrm{mGy} \mathrm{cm}$.

Using scanner \#1, non-contrast head CT was performed with either a conventional protocol $(120 \mathrm{kVp}, 300 \mathrm{mAs}$, a rotation time of $1.0 \mathrm{~s}$, and a slice thickness of $4 \mathrm{~mm}$ for the skull base and $8 \mathrm{~mm}$ at the supratentorial level) or a helical protocol $(120 \mathrm{kVp}, 300 \mathrm{mAs}$, pitch of 0.625 , reconstructed slice thickness of $3 \mathrm{~mm}$ ). The helical protocol was used if sagittal or coronal reconstruction was required, or if the patient was likely to find it difficult to stay still during the examination. The DLPs obtained with scanner \#1 (Fig. 1) and the typical conventional protocol were reasonably low $\left(\mathrm{CTDI}_{\mathrm{vol}}=82.4\right.$ (supratentorial)/115.8 $\mathrm{mGy}$ (skull base); however, that obtained with the helical scan protocol $\left(\mathrm{CTDI}_{\mathrm{vol}}=93.4 \mathrm{mGy}\right)$ exceeded the DRL. Based on these results, we decided to develop a new imaging protocol for scanner \#1 to replace the previous helical protocol, with the capability of sagittal and coronal image reconstruction.

Table 1 Summary of the non-contrast adult head CT scans performed at our institution during the 3-month period

\begin{tabular}{lll}
\hline $\begin{array}{l}\text { Scanner \# } \\
\text { performed by the scanner }\end{array}$ & Mean DLP (mGy cm) \\
\hline 1 & $1842(90.3 \%)$ & 1401.2 \\
2 & $123(6.0 \%)$ & 1123.9 \\
3 & $47(2.3 \%)$ & 1383.0 \\
4 & $28(1.4 \%)$ & 1123.1 \\
\hline
\end{tabular}

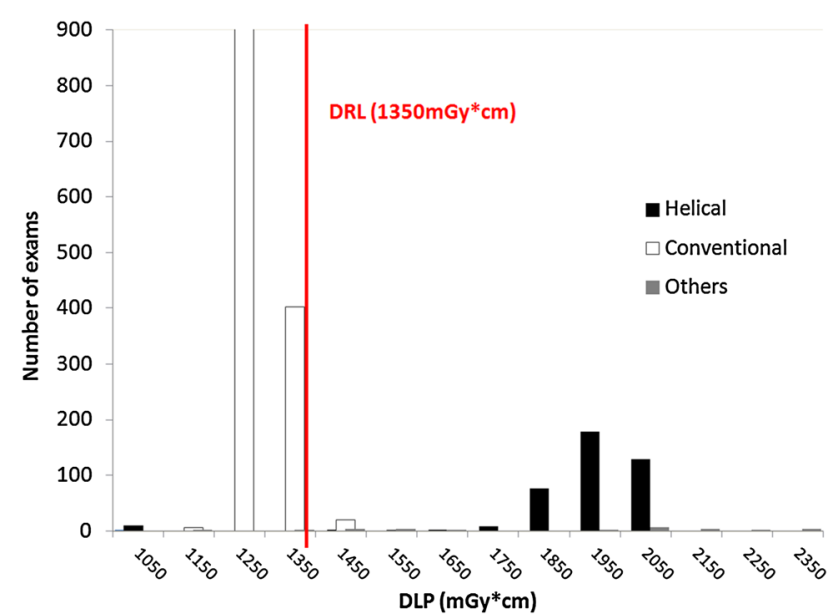

Fig. 1 Distribution of the DLP for non-contrast adult head CTs performed by scanner 1, stratified based on the protocol. Others include examinations where the head was scanned twice or more due to motion artifact etc 


\section{Development of the new protocol based on the phantom study}

The results of the phantom study, including those for the background noise and CNR, are shown in Fig. 2 and Table 2. Radiation exposures obtained with the three candidate protocols and the protocols that were currently in use were determined. As scan techniques with revised helical scanning parameters did not achieve an adequate CNR (data not shown), we selected wide-volume scan protocols capable of reconstruction in the coronal and sagittal planes as candidate scans. The smallest circle of the $0.3 \%$ contrast supraslice test object identifiable by both the staff radiologist (K.K.) and the senior CT technologist (Y.K.) had a diameter of $8 \mathrm{~mm}$ for all six of the evaluated protocols. The limiting spatial resolution was approximately 10 line pairs $/ \mathrm{cm}$ for all protocols. Among the candidate protocols, we considered that protocol \#6 was the most suitable, because the CNR was the highest (scan parameters of 120 $\mathrm{kVp}$ and $350 \mathrm{mAs}$, a wide-volume scan using a $4-\mathrm{cm}$ detector, and a reconstructed slice thickness of $5 \mathrm{~mm}$, CTDIvol $=69.8 \mathrm{mGy}$ ) (Fig. 2; Table 2).

Although the phantom study confirmed that image quality was similar for the protocols that were currently being applied and the candidate protocols, we decided that the new protocol should not be used for patients who had never undergone a non-contrast head CT at our institution. The new protocol could also be applied to other patients provided this was confirmed and agreed upon by our radiologists and the referring physicians, and it did not have adverse effects on image assessment. Therefore, the initial subjects in the new protocol were patients undergoing a repeat/follow-up head CT examination with coronal or sagittal reconstruction for the helical scan. Because widevolume scans are more susceptible to motion artifacts than helical scans, we continued to use the established helical scan protocol for patients who found it difficult to stay still during the examination.

\section{Feedback from referring physicians and diagnostic radiologists}

After implementing the new imaging protocol on 1st December 2015, we received feedback from our radiologists. All of the comments were related to minor technical errors during reconstruction, such as a lack of bone windows in the reconstruction image, and an error in the setting of the correct slice thickness. The referring physicians did not send any negative feedback responses regarding image quality. Representative non-contrast head CT images obtained with the helical and new protocols are presented in Fig. 3.

\section{Post-implementation analyses}

Among a total of 1680 non-contrast adult head CT exams performed during the 2-month period after its

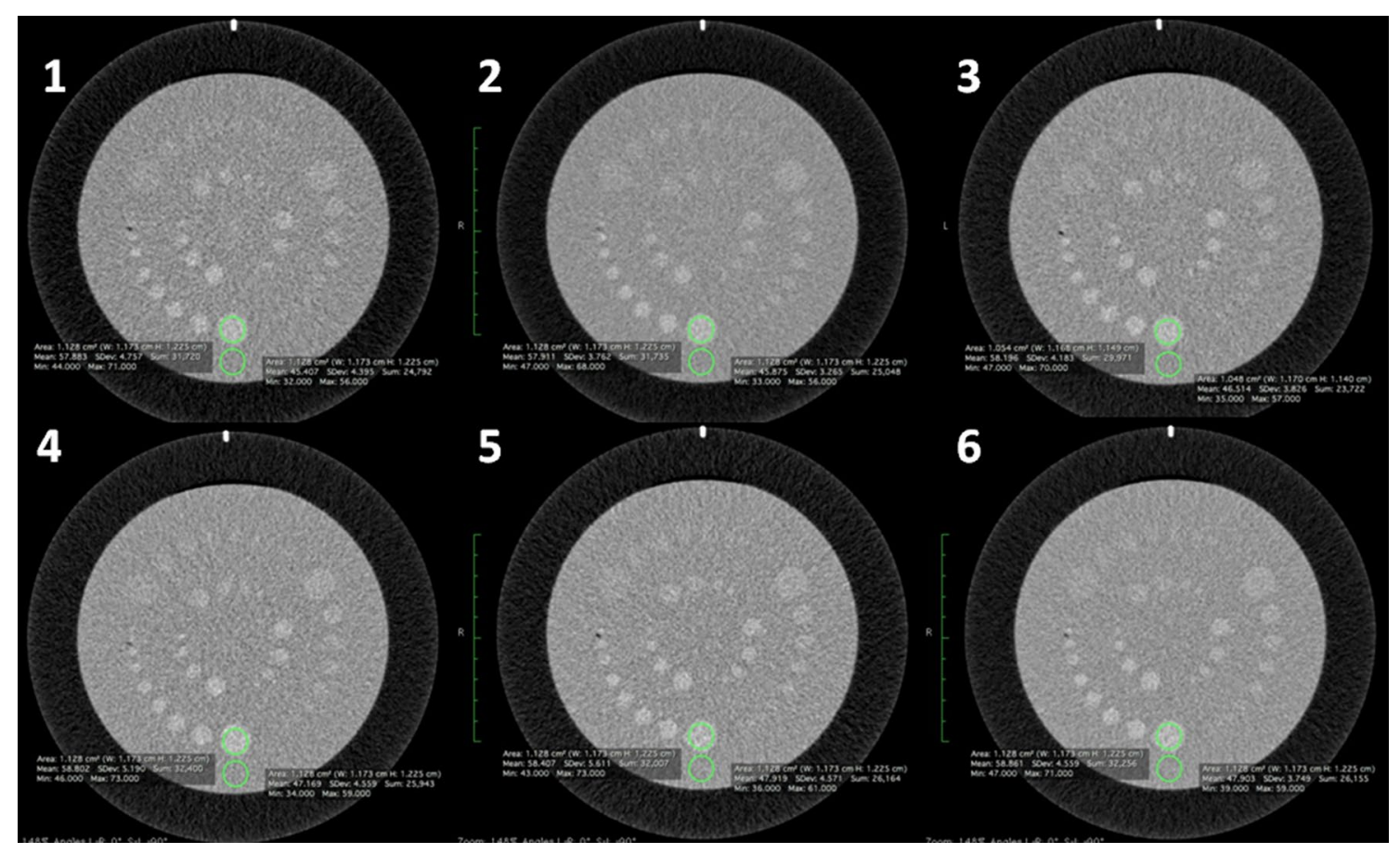

Fig. 2 Low-contrast phantom (CTP515) evaluation with region of interest (ROI) placement. Protocol numbers correspond to those in Table 2 
Table 2 Comparison of the candidate scan protocols with the scan protocols that were currently being applied

\begin{tabular}{|c|c|c|c|c|c|c|}
\hline \multirow[t]{3}{*}{ Scan protocol } & \multicolumn{3}{|c|}{ Protocols that were currently being applied } & \multicolumn{3}{|c|}{ Candidate protocols } \\
\hline & 1 & 2 & 3 & 4 & 5 & 6 \\
\hline & $\begin{array}{l}\text { Conventional } \\
\text { (skull base) }\end{array}$ & $\begin{array}{l}\text { Conventional } \\
\text { (supratentorial) }\end{array}$ & Helical & Wide volume & Wide volume & Wide volume \\
\hline Tube voltage $(\mathrm{kV})$ & 120 & 120 & 120 & 120 & 120 & 120 \\
\hline Tube current (mA) & 200 & 200 & 300 & 350 & 350 & 350 \\
\hline Rotation time (second) & 1.5 & 1.5 & 1.0 & 0.75 & 1.0 & 1.0 \\
\hline Slice collimation & $1 \mathrm{~mm} \times 4$ & $2 \mathrm{~mm} \times 4$ & $1 \mathrm{~mm} \times 40$ & $1 \mathrm{~mm} \times 40$ & $1 \mathrm{~mm} \times 40$ & $1 \mathrm{~mm} \times 40$ \\
\hline Pitch factor & - & - & 0.625 & - & - & - \\
\hline Reconstructed slice thickness & $4 \mathrm{~mm}$ & $8 \mathrm{~mm}$ & $3 \mathrm{~mm}$ & $5 \mathrm{~mm}$ & $3 \mathrm{~mm}$ & $5 \mathrm{~mm}$ \\
\hline $\mathrm{CTDI}_{\mathrm{vol}}(\mathrm{mGy})$ & 115.8 & 82.4 & 93.4 & 53 & 69.8 & \\
\hline Craniocaudal range $(\mathrm{mm})$ & 136 & & 150 & 151 & 151 & 151 \\
\hline DLP (mGy cm) & 1253.8 & & 1718.6 & 801 & 1053.2 & \\
\hline Background SD & 4.4 & 3.3 & 3.8 & 4.6 & 4.6 & 3.7 \\
\hline $\mathrm{CNR}$ & 2.8 & 3.6 & 3.1 & 2.5 & 2.3 & 3.0 \\
\hline
\end{tabular}

$C T D I C T$ dose index, $D L P$ dose length product, $S D$ standard deviation, $C N R$ contrast to noise ratio

Fig. 3 Images from a 74-yearold man who had normal pressure hydrocephalus and thus required repeat CT studies with coronal reconstruction. a, b Helical scan with DLP of $2045.4 \mathrm{mGy} \mathrm{cm}$. c, d New protocol (volume scan) with DLP of $1053.2 \mathrm{mGy} \mathrm{cm}$, showing comparable image quality with even fewer streak artifacts

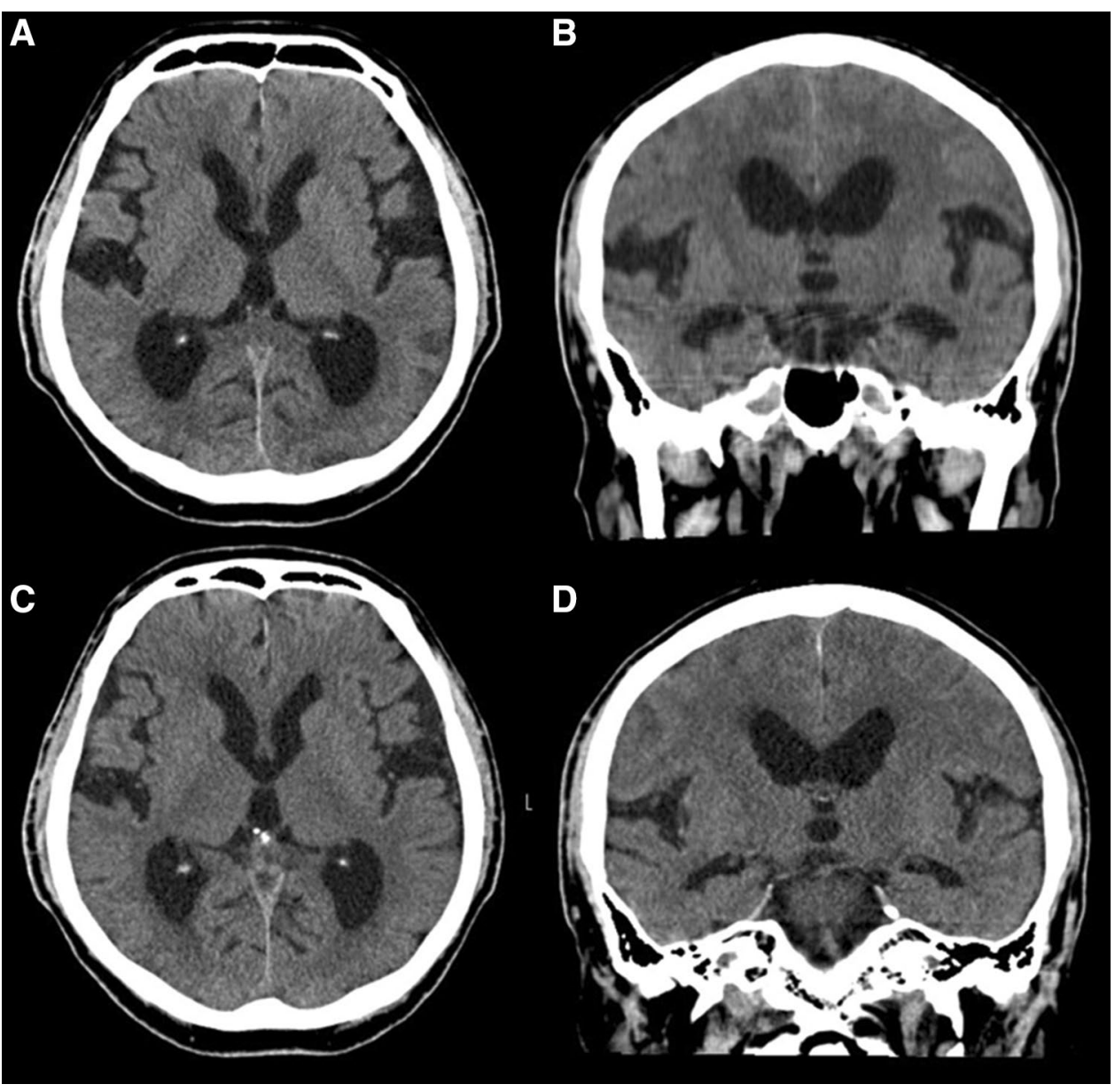


implementation, the new protocol was used in $23.3 \%$ (391/1680). The overall mean DLP after implementing the new protocol was $1365 \mathrm{mGy} \mathrm{cm}$, a significant decrease compared to the previously applied protocol $(p<0.01)$ (Fig. 4). When we excluded examinations of patients who were scanned twice or three times due to inadequate image quality, including motion artifact, the mean DLP was $1348 \mathrm{mGy} \mathrm{cm}$. No significant decrease was observed in the prevalence of cases with significant findings (categories 3 or 4) following implementation of the new protocol (Fig. 5). Subjective image quality scores evaluated in 30 CT scans were comparable before and after implementation of the new imaging protocol (Table 3).

\section{Discussion}

As it is common for patients with complex or chronic diseases to undergo multiple head CT studies, radiation dose optimization is important. In the present paper, we have described how we developed and implemented a new low-dose CT protocol and compared its mean DLP to the relevant currently established DRL in Japan. Although further follow-up is necessary to evaluate the clinical impact of the new protocol, there is currently no evidence to suggest that the new protocol adversely affects clinical interpretation. The new protocol results in a significant reduction in radiation exposure. We are still using
Fig. 4 Variation in the mean dose length product during the period from a few months before the implementation of the new protocol (on 1st December) to a few months after it

Fig. 5 Prevalence of each class before and after the implementation of the new protocol
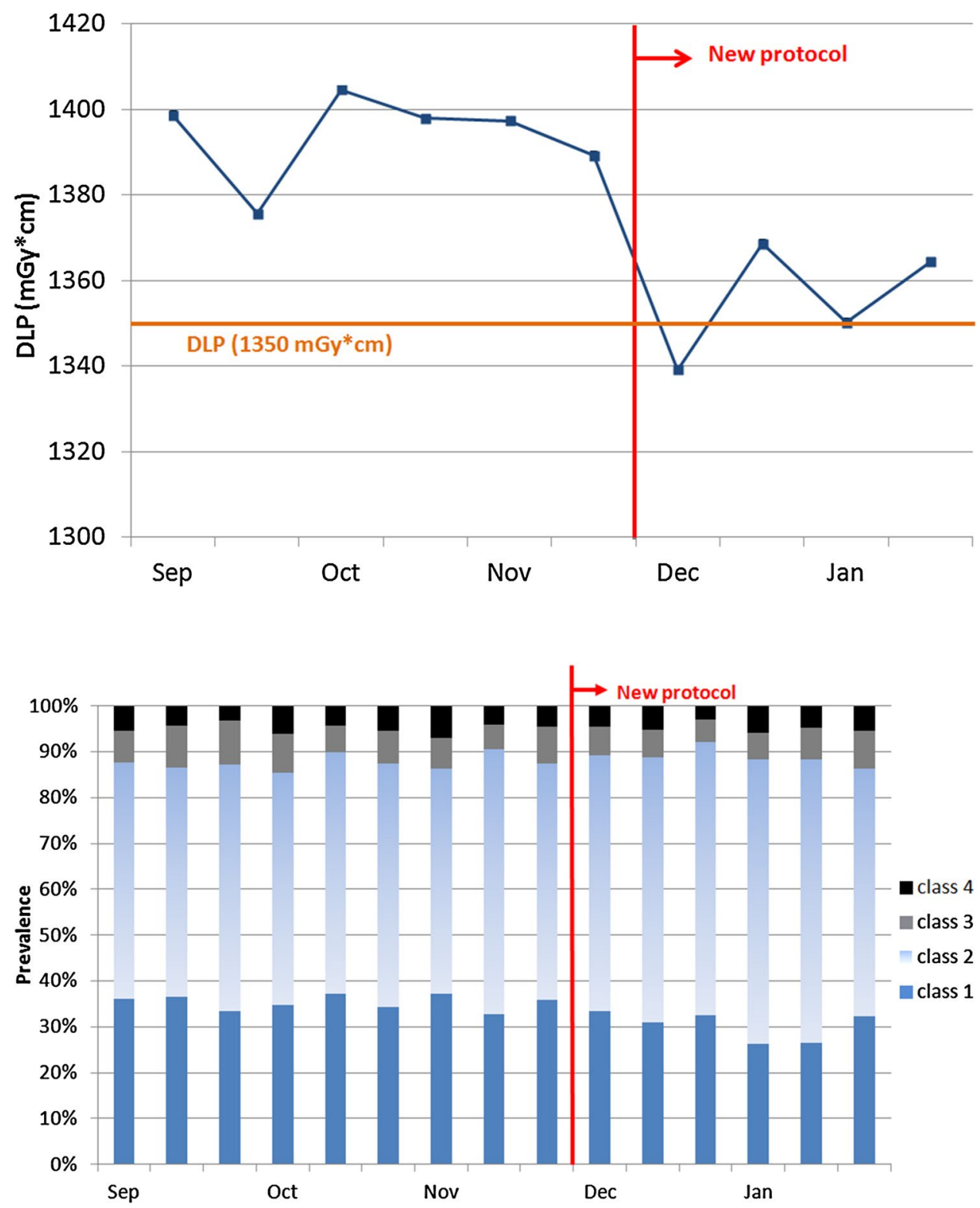
Table 3 Comparison of the subjective image quality scores achieved using the new and old scan protocols

\begin{tabular}{|c|c|c|c|c|c|c|}
\hline & \multicolumn{3}{|l|}{ Reader 1} & \multicolumn{3}{|l|}{ Reader 2} \\
\hline & Old & New & $p$ value & Old & New & $p$ value \\
\hline Artifact & $2.33(5)$ & $2.40(6)$ & 0.71 & $2.40(6)$ & $2.47(7)$ & 0.72 \\
\hline Noise & 2.27 (4) & $2.40(6)$ & 0.45 & $2.33(5)$ & $2.47(7)$ & 0.46 \\
\hline Gray-white matter differentiation & $2.40(6)$ & $2.53(8)$ & 0.47 & $2.47(7)$ & $2.53(8)$ & 0.72 \\
\hline
\end{tabular}

* Mean values are shown, with the number of exams scored as 3 out of 15 CTs indicated inside the parentheses the new protocol routinely, while continuous reviewing and periodic evaluation of the protocols are required.

The Japanese DRL for non-contrast adult head CT ( $85 \mathrm{mGy}_{\text {for }} \mathrm{CTDI}_{\mathrm{vol}}$ and $1350 \mathrm{mGy} \mathrm{cm}$ for DLP) is slightly higher than those adopted in other countries [2], probably because the imaging parameters are selected to detect the early CT signs of ischemic stroke in many Japanese institutions, so low contrast resolution is needed at the expense of radiation exposure. In the United States, after establishing a DRL of $60 \mathrm{mGy}$ for adult head CTDIw in 2002, the American College of Radiology (ACR) received complaints that head examination image quality was inadequate at a CTDIw value of $<60 \mathrm{mGy}$ [6]. Therefore, the ACR increased the DRL to $75 \mathrm{mGy}$ in the 2008 revision [6]. This reminds us that it is important to balance the potential benefits of a low-dose technique with the risk of low image quality. While high image quality is important when ischemic stroke is suspected, a quality-oriented protocol may not be justified for other clinical scenarios if the aim of follow-up or repeat studies is to identify complications or gross morphologic changes such as bleeding or to assess ventricular size [7]. Thus, follow-up or repeat head CT is generally tolerable with a slight degradation in image quality. At our institution, the helical scan protocol was mainly used when coronal or sagittal reconstructions were necessary (e.g., when following up normal pressure hydrocephalus). In such cases, slightly lower image quality is perfectly adequate, but our phantom study confirmed that the image quality of the new protocol was similar. Although the current Japanese DRL for non-contrast adult head CT is slightly higher than those used in other countries, we expect that the radiation dose for non-contrast head CT will be reduced in many Japanese institutions, just as we did after the Japanese DRL was established, and the next version of the Japanese DRL based on another nationwide survey will lower the reference level.

To confirm that the new protocol did not have a negative impact on clinical interpretation, we analyzed the prevalence of significant findings. However, this may not be feasible without the personnel to perform such analyses. In this case, objective and subjective evaluations of image quality in sample cases in addition to feedback from the diagnostic radiologists and the referring physicians after implementation of the new imaging protocol are useful for assessing the clinical impact of the new protocol.
Because the new protocol is intended for routine clinical use rather than for research, it is important that CT technologists of any skill level can easily adhere to the protocol. There was some initial confusion when we first implemented the new protocol, especially during night shifts. Further instruction and a hands-on training session by senior technologists were effective in this regard, but the most important policy was that the protocol should not be too complicated. We adopted a wide-volume scan for the new protocol, but the hardware and software that we used are not available at all institutions, so our protocol may not be applicable to other institutions. Nevertheless, we expect that the description of our review of the protocols, data analysis, and post-implementation evaluation could also be utilized by those seeking to optimize radiation exposure in routine CT examinations.

In summary, this report describes the implementation of a new imaging protocol with a reduced radiation dose for non-contrast head CT imaging, where the currently established Japanese DRLs were employed as the reference.

\section{References}

1. International Commission on Radiological Protection. Radiological protection and safety in medicine. A report of the International Commission on Radiological Protection. Ann ICRP. 1996;26:1-47.

2. Japan Association on Radiological Protection in Medicine, et al. Diagnostic reference levels based on latest surveys in Japan: Japan DRLs 2015. 2015. http://www.radher.jp/J-RIME/report/ DRLhoukokusyoEng.pdf.

3. Nishizawa K, Matsumoto M, Iwai K, Maruyama T. Survey of CT practice in Japan and collective effective dose estimation. Nihon Igaku Hoshasen Gakkai Zasshi Nippon Acta Radiologica. 2004;64:151-8.

4. Tsushima Y, Taketomi-Takahashi A, Takei H, Otake H, Endo K. Radiation exposure from CT examinations in Japan. BMC Med Imaging. 2010;10:24.

5. The Phantom Laboratory. Catphan 500 and 600 manual. 2006. http://www.uio.no/studier/emner/matnat/fys/FYS4760/h07/Catphan500-600manual.pdf.

6. McCollough C, Branham T, Herlihy V, Bhargavan M, Robbins L, Bush K, McNitt-Gray M, Payne JT, Ruckdeschel T, Pfeiffer D, Cody D, Zeman R. Diagnostic reference levels from the ACR CT Accreditation Program. J Am Coll Radiol. 2011;8(11):795-803.

7. Cohnen M, Fischer H, Hamacher J, Lins E, Kotter R, Modder U. $\mathrm{CT}$ of the head by use of reduced current and kilovoltage: relationship between image quality and dose reduction. AJNR Am J Neuroradiol. 2000;21:1654-60. 